\title{
Up to seven planets orbiting HD 10180: probing the architecture of low-mass planetary systems
}

\author{
C. Lovis ${ }^{1}$ and the HARPS team \\ ${ }^{1}$ Observatoire Astronomique de l'Université de Genève, 51 Chemin des \\ Maillettes, 1290 Sauverny, Switzerland [Christophe.Lovis@unige.ch]
}

We are conducting a high-precision radial velocity survey with the HARPS spectrograph which aims at characterizing the population of ice giants and super-Earths around nearby solar-type stars. This will lead to a better understanding of their formation and evolution, and yield a global picture of planetary systems from gas giants down to telluric planets. Progress has been possible in this field thanks in particular to the sub-m.s ${ }^{-1}$ radial velocity precision achieved by HARPS. We present here new high-quality measurements from this instrument. We report the discovery of a planetary system comprising at least five Neptune-like planets with minimum masses ranging from 12 to $25 \mathrm{M}_{\text {Earth }}$, orbiting the solar-type star HD 10180 at separations between 0.06 and 1.4 AU. A sixth radial velocity signal is present at a longer period, probably due to a $65-\mathrm{M}_{\text {Earth }}$ object. Moreover, another body with a minimum mass as low as $1.4 \mathrm{M}_{\text {Earth }}$ may be present at $0.02 \mathrm{AU}$ from the star. This is the most populated exoplanetary system known to date. The planets are in a dense but still well-separated configuration, with significant secular interactions. Some of the orbital period ratios are fairly close to integer or half-integer values, but the system does not exhibit any mean-motion resonances. General relativity effects and tidal dissipation play an important role to stabilize the innermost planet and the system as a whole. Numerical integrations show long-term dynamical stability provided true masses are within a factor $\sim 3$ from minimum masses. We further note that several low-mass planetary systems exhibit a rather "packed" orbital architecture with little or no space left for additional planets. In several cases, semi-major axes are fairly regularly spaced on a logarithmic scale, giving rise to approximate Titius-Bode-like (i.e. exponential) laws. These dynamical architectures can be interpreted as the signature of formation scenarios where type I migration and interactions between protoplanets play a major role. However, it remains challenging to explain the presence of so many Neptunes and super-Earths on non-resonant, well-ordered orbits within 1-2 AU of the central star. Finally, we also confirm the marked dependence of planet formation on both metallicity and stellar mass. Very massive systems are all found around metal-rich stars more massive than the Sun, while low-mass systems are only found around metal-deficient stars less massive than the Sun.

\section{References}

Lovis C., Ségransan D., Mayor M., et al. 2010, A\&A in press, arXiv:1011.4994

This is an Open Access article distributed under the terms of the Creative Commons Attribution-Noncommercial License 3.0, which permits unrestricted use, distribution, and reproduction in any noncommercial medium, provided the original work is properly cited. 


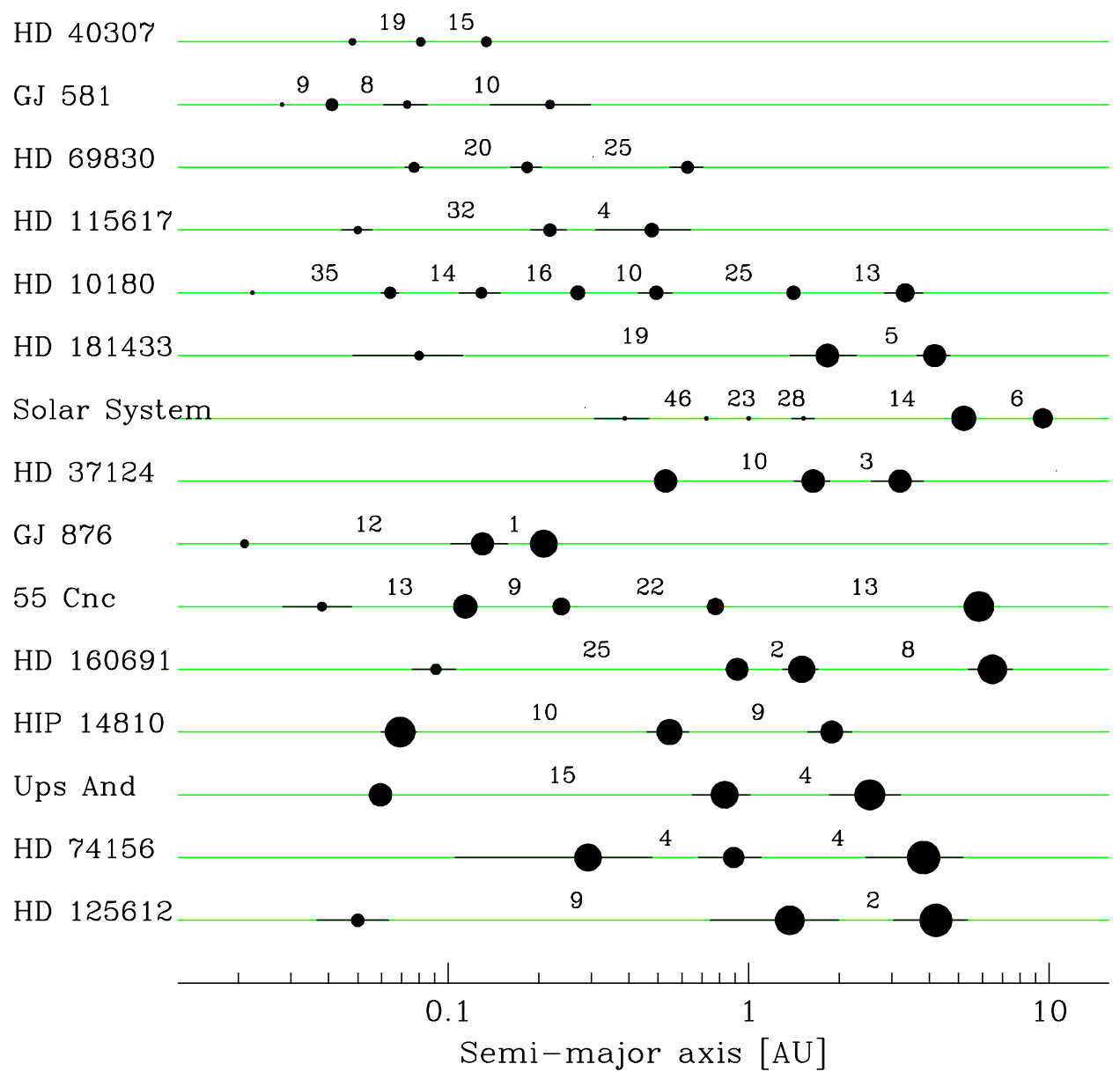

Figure 1: The 15 planetary systems with at least three known planets as of May 2010. The numbers give the minimal distance between adjacent planets expressed in mutual Hill radii. Planet sizes are proportional to $\log (\mathrm{m} \operatorname{sini})$. 